\title{
Study Day
}

National Cancer Institute

\section{Source}

National Cancer Institute. Study Day. NCI Thesaurus. Code C82437.

The day that an examination, assessment, or treatment is performed. 